\title{
Linx
}

Revue des linguistes de l'université Paris X Nanterre

$44 \mid 2001$

Spécificité et histoire des discours sémiotiques

\section{Sémiotique et sciences de la culture}

\section{François Rastier}

\section{OpenEdition}

Journals

Édition électronique

URL : http://journals.openedition.org/linx/1058

DOI : 10.4000/linx.1058

ISSN : 2118-9692

\section{Éditeur}

Presses universitaires de Paris Nanterre

\section{Édition imprimée}

Date de publication : 1 juin 2001

Pagination : 149-168

ISSN : 0246-8743

\section{Référence électronique}

François Rastier, «Sémiotique et sciences de la culture », Linx [En ligne], 44 | 2001, mis en ligne le 05 juillet 2012, consulté le 30 avril 2019. URL : http://journals.openedition.org/linx/1058 ; DOI : 10.4000/ linx.1058

Ce document a été généré automatiquement le 30 avril 2019.

Département de Sciences du langage, Université Paris Ouest 


\title{
Sémiotique et sciences de la culture
}

\author{
François Rastier
}

Seule une discipline de l'interdisciplinarité peut

convenir à l'interprétation des phénomènes

humains

G. Agamben, Stanze

1 La sémiotique semble encore une discipline sans frontières; nous l'aborderons donc par restriction pour la questionner sur le texte. Les réponses partielles que nous obtiendrons nous conduiront à poser d'autres questions, d'ordre épistémologique.

2 Nous considérons la linguistique comme la sémiotique des langues et des textes, mais puisque la sémiotique et la linguistique ont connu des destins séparés, il nous faudra interroger leurs rapports.

\section{Questions ouvertes}

\section{La sémiotique est-elle une discipline?}

Cette question préjudicielle mérite encore d'être posée, car tout corps de théories n'est pas appelé à devenir une discipline. L'essor des universités au XIX ${ }^{\mathrm{e}}$ s'est accompagné par une disciplinarisation des savoirs savants, mais la sémiotique ne s'est pas (encore) disciplinarisée. Faute sans doute d'une collectivité suffisamment unifiée pour se pourvoir de critères d'évaluation, elle n'a pu définir ni critères de qualité reconnus, ni cursus de formation. De fait, les rares enseignements de sémiotique dépendent de départements de linguistique, plus rarement encore de philosophie. Plus récemment, les sciences de la communication ont accueilli des enseignements de sémiotique. Bien que la demande sociale aille croissant, il n'est pas encore certain que les sciences de la communication, qui sont plutôt, de fait, des techniques, aient l'assise épistémologique nécessaire au développement d'une sémiotique fondamentale. Ces questions académiques restent ouvertes, et si la sémiotique parvient à se disciplinariser, ce succès académique ne sera pas pour autant la preuve du bien-fondé épistémologique de son autonomisation. 
Corps de savoirs, de postulats et de conjectures, la sémiotique est issue de deux disciplines : la philosophie (notamment la philosophie du langage) depuis Locke, qui a sémiotisé la tradition de la logique philosophique, et la linguistique depuis Saussure, Hjelmslev, Greimas notamment. Bien qu'elles n'aient pas le même statut, ni les mêmes objectifs, elles peuvent dialoguer en restant sur le terrain philosophique, dans la mesure où la sémantique, domaine charnière entre linguistique et philosophie du langage, reste largement ouverte à des questions métaphysiques comme celle de la référence. Mais ce dialogue reste peu fructueux, et les mêmes arguments y reviennent depuis des siècles (cf. Eco 1989).

Malgré les efforts de conciliation, la synthèse reste impossible, car la sémiotique issue de la linguistique et la sémiotique philosophique demeurent séparées par deux questions.

6 En premier lieu, elles divergent sur la question des seuils sémiotiques qui séparent les langages et autres systèmes de signes des autres niveaux de la réalité : le niveau physique et le niveau des (re)présentations. Là où la linguistique propose un seuil qui passe par les systèmes de signes (biplanes selon la proposition de Hjelmslev), la philosophie du langage, dans sa tradition intentionnaliste de souche augustinienne milite pour un abaissement voire une suppression des seuils. Pour une conscience, tout peut signifier, dans la mesure où l'intentionnalité sémiotise tout ce qu'elle vise (cf. en philosophie analytique Pierre Jacob, Pourquoi les choses ont une signification). Mieux, pour Peirce, la sémiotique intéresse tant les animaux que les plantes et les minéraux ${ }^{1}$; et des sémioticiens peirciens contemporains ont parlé très sérieusement de sémiotiques des particules élémentaires, hadronique, leptonique, etc.

7 Se recommandant pourtant de Saussure et de Hjelmslev, Greimas de son côté n'a pas hésité à formuler l'énigmatique programme d'une "sémiotique du monde naturel» (1970); et bizarrement, il a été suivi sur ce point. Nous nous cantonnerons pour notre part au monde culturel.

8 Sémiotique philosophique et sémiotique linguistique divergent sur une seconde question critériale : leur objet comprend-il deux ordres de réalité ? Là encore la conception de la signification reste déterminante. Pour la tradition de la sémiotique philosophique, tant aristotélicienne qu'augustinienne, le réalisme l'emporte, et avec lui le postulat réaliste que la signification est un rapport entre un concept et un objet. Elle doit donc postuler à toute force une ontologie des objets.

9 De tradition occamiste, le prétendu nominalisme de Locke et de la philosophie analytique n'est qu'un réalisme des individus, alors que la sémantique saussurienne milite en revanche pour un non-réalisme de principe. Son refus de l'ontologie revêt une valeur fondatrice pour distinguer la sémantique des langues de celle du langage, détenue depuis Aristote par la logique. L'ontologie le cède alors à une dé-ontologie, et la conception représentationnelle, théorétique, de la signification, à une conception praxéologique, qui la définit comme sens de performances sémiotiques au sein de pratiques sociales (cf. l'auteur 1996a).

10 La linguistique a pu devenir le noyau constituant de la sémiotique contemporaine par son projet scientifique (qui la séparait de la philosophie), par son épistémologie et sa méthodologie de science historique et comparée. Cependant, cette évolution n'a pas été comprise, et la linguistique a souvent été exploitée et supplantée d'un même mouvement par des projets énonciatifs ou cognitifs qui entendaient la dépasser. Catégories 
universelles, sujets transcendentaux sont restés la matière ordinaire et passablement spéculative de la réflexion.

11 Cependant, on ne peut fonder la sémiotique par abstraction. Par exemple, ce n'est pas l'abstraction de formes à partir des langues qui permet de décrire les fonctionnement propres aux images où aux musiques: cette abstraction ne peut retrouver que le fonds métaphysique de la tradition logico-grammaticale qui privilégie un petit nombre de relations et de catégories, combinées en modèles constitutionnels, beaucoup trop puissants, par leur trivialité même, et que l'on peut projeter sur des objets quelconques, certes sans grand effort, mais sans gain descriptif notable. Dès lors que l'on cesse de postuler divers universaux ethnocentriques et logocentriques qu'on lui donne pour organon, voire pour objet, la sémiotique ne peut être que fédérative : l'iconologie, la musicologie, la linguistique, la chorégraphie, etc. sont bien entendu parties prenantes de cette fédération.

12 Conformément au projet saussurien, la création de la sémiotique à partir de la linguistique s'autorise du caractère exemplaire de cette discipline, qui occupe une place importante au sein de la fédération des sciences de la culture - nous développerons ce point à la fin de cette étude.

\section{La sémiotique est-elle une science des signes?}

13 La sémiotique contemporaine a peut-être défini trop restrictivement son objet. Elle se présente en effet comme une science des signes, et bien des ouvrages de sémiotique sont consacrés à la typologie des signes (cf. Eco 1992). Dans la tradition anglo-saxonne, la définition peircienne de la sémiotique comme «doctrine des signes » (1956: I-98) a une grande autorité, et Thomas Sebeok, qui préside depuis trente ans aux destinées académiques mondiales de la sémiotique, souligne que le concept clé de la sémiotique demeure toujours le signe. Pap estimait naguère résumer l'opinion de tout sémioticien en réaffirmant que la sémiotique est l'étude des signes.

14 L'affaire serait entendue si le signe n'était un artefact des sémioticiens : d'une part, son identification est le résultat d'une interprétation, non son point de départ; d'autre part, en règle générale, les pratiques sémiotiques ne mettent pas en œuvre des signes isolés, mais des formations complexes dont la segmentation est toujours problématique, parfois impossible. La définition de la sémiotique comme science des signes s'inscrit alors dans la tradition logique et grammaticale, d'ascendance aristotélicienne, ordinaire dans cette discipline. Universaliste, statique, réaliste, elle s'appuie sur une ontologie des substances, et subordonne donc le signe au concept: il semble bien que la solitude du signe soit la rançon de l'autarcie du concept.

15 Certes, les courants sémiotiques issus de la linguistique plutôt que de la logique ou de la grammaire soulignent que la sémiotique prend pour objet les systèmes de signes. C'est le cas, dans la tradition européenne, de Saussure et Hjelmslev, tout comme de l'École de Tartu, chez Ivanov, Lotman, Lekomcev notamment. Cependant, les systèmes de signes sont ordinairement conçus comme des syntaxes : par exemple, la théorie de Hjelmslev étend des procédures d'analyse morphosyntaxique à l'ensemble des systèmes de signes. Or, même pour les langues, cette conception syntaxique ne convient pas, ou fort mal. Dans l'analyse des textes, on relève toutes sortes d'unités qui ne consistent pas en signes, comme les thèmes ou les fonctions narratives. Les signes sont les unités les moins complexes, cela n'entraîne pas qu'elles soient fondamentales, au sens où toutes les autres 
se réduiraient à elles sans reste ${ }^{2}$. Enfin, une langue ne consiste pas en un et un seul système de signes, dans la mesure où tout texte témoigne de l'interaction de plusieurs sortes de systèmes, notamment de normes. C'est pourquoi aucune grammaire ne peut engendrer un texte; et faute de tenir compte des normes, celles qui peuvent engendrer des phrases ne peuvent écarter les phrases indicibles - ou non dicibles parce qu'inacceptables selon les canons de la rationalité.

\section{La sémantique des textes et ses propositions pour la sémiotique}

\section{Le sens et la sémiosis textuelle}

16 Une autre opposition intéresse les paliers de la description : on parle alors de la signification d'un mot et du sens d'un texte. Cette seconde distinction reflète alors la distinction entre les deux problématiques logico-grammaticale et herméneutique / rhétorique. Bizarrement, la plupart des théories de la signification en restent au signe isolé, bien qu'il soit un artefact : il n'est pas observé empiriquement et seule une décision méthodologique permet d'isoler un signe. En revanche, les énoncés empiriques sont des textes oraux ou écrits, ou des passages de ces textes ${ }^{3}$.

Bien qu'elle occupe une position intermédiaire entre le signe et le texte, la phrase est traditionnellement conçue à partir du signe et non du texte. Le recours de plus en plus fréquent au contexte reste ambigu, car c'est une zone d'extension, relativement au signe et à la phrase, mais une zone de restriction, relativement au texte.

La sémiosis, relation fondamentale qui unit les deux faces du signe, doit être rapportée aux deux plans du contenu et de l'expression des textes et des autres performances sémiotiques, et non plus définie comme une relation entre le signifiant et le signifié du signe. D'autre part, elle ne peut être définie par une relation logique simplement formulable, comme l'inférence dans la tradition intentionnaliste ou la présupposition réciproque dans la tradition structuraliste. Enfin, le signifiant n'en est pas le point de départ, malgré les théories inférentielles ou associationnistes, car il a lui-même à être reconnu.

19 En d'autres termes, les relations qui établissent le sens vont de signifié en signifié, aussi bien que du signifié vers le signifiant. Aussi, nous définissons la sémiosis à partir du réseau des relations entre signifiés au sein du texte - en considérant les signifiants comme des interprétants qui permettent de construire certaines de ces relations. Nous concevons ces relations comme des parcours orientés. On pourrait distinguer sans doute autant de sortes de sémiosis que de sortes de parcours élémentaires, mais il faut souligner que tous les signes linguistiques ne se prêtent pas aux mêmes parcours.

20 Enfin, la sémiosis ne peut être fixée que comme résultat de l'interprétation, non comme son départ. L'identification des signifiants semble un des points d'entrée dans le parcours interprétatif, mais elle est précédée par les attentes et présomptions que définissent le contrat propre au genre textuel de la pratique en cours ; aussi semble-t-elle également un point de retour.

21 Redéfinir ainsi la sémiosis la rapporte nécessairement au concept de parcours interprétatif. Le sens n'est plus fixé par un codage préalable qui associerait strictement un signifiant et un signifié ou une classe de signifiés (car la langue n'est pas une nomenclature) : il est 
produit dans des parcours qui discrétisent et unissent des signifiés entre eux, en passant par des signifiants.

Les genres, dans la mesure où ils déterminent au palier textuel les modes de corrélation entre les plans du signifié et du signifiant, sont les facteurs déterminants de la sémiosis textuelle. Ils contraignent non seulement le mode mimétique du texte, mais aussi ses modes de production et d'interprétation. Ils témoignent par ailleurs du caractère instituant des pratiques sociales dans lesquelles ils prennent place.

\section{Pour une refondation interprétative de la sémiotique}

Venons-en à une question cruciale et souvent omise ; comment concevoir l'unité des deux plans du langage, mixte jugé intolérable de sensible et d'intelligible? On peut bien entendu proposer une réponse fonctionnelle, et rappeler que le langage a de fait sinon par vocation une fonction médiatrice entre ces deux sphères ${ }^{4}$. Il faut encore que cette conception même fasse droit à l'unité des deux plans du langage. Une conception non dualiste se doit en effet d'intégrer signifiants et signifiés dans les mêmes parcours : ils sont discrétisés d'ailleurs par les mêmes types d'opérations ${ }^{5}$, et les signifiants ne sont pas plus « donnés » que les signifiés. La théorie des parcours interprétatifs permet de rendre compte du lien problématique entre les deux plans du langage: ainsi, la sémantique interprétative a maintes fois souligné que l'actualisation de traits sémantiques exigeait le passage par ces interprétants que sont selon elle les signifiants (par exemple, la rime est ordinairement l'indice d'une relation sémantique entre sémèmes).

Toutes ces propositions convergent vers une refondation interprétative de la sémantique, et au-delà, de la sémiotique, à partir de thèses qui intéressent le palier du signe et celui du texte.

- (i) Un signe n'est qualifié comme tel que par un parcours interprétatif. Par exemple, un signe de ponctuation considéré comme une simple démarcation du signifiant peut être sémantisé en contexte, et fonctionner comme un morphème (un point d'exclamation peut signifier 'brusquerie' par exemple).

- (ii) Aucun signe n'est par lui-même référentiel, inférentiel ou différentiel. Ces relations sont privilégiées par diverses théories, mais les parcours interprétatifs effectifs sont plus complexes, et leur analyse ne permet pas de retrouver des relations simplement qualifiables (par exemple, les inférences interprétatives ne sont pas formelles); ils sont sans doute plus près des processus perceptifs de la reconnaissance de formes que du calcul.

- (iii) Le texte, ou la performance sémiotique, est l'unité fondamentale pour la problématique rhétorique/herméneutique. Il faut cependant se garder de confondre, comme le fait la problématique logico-grammaticale depuis les Stoïciens, le fondamental et l'élémentaire : si par exemple le signe linguistique (morphème) est une unité minimale, elle n'est pas pour autant fondamentale.

Si pour la problématique rhétorique / herméneutique le texte est l'unité fondamentale, l'unité linguistique maximale est le corpus de référence. Cette expression appelle deux précisions : le corpus dépend du point de vue qui a présidé à sa constitution - qu'il s'agisse de limites contigentes comme celles d'une histoire conversationnelle, ou réfléchies comme celles d'un corpus textuel à l'intérieur d'un genre. La référence s'entend ici dans l'acception philologique - et non dans l'acception logique, car on ne réfère jamais qu'à une doxa, c'està-dire un ensemble d'axiomes normatifs localement établis par le corpus des textes oraux ou écrits faisant autorité dans la pratique en cours ${ }^{6}$. 
- (iv) Ces formes d'incidence se composent, et l'on pourrait dire que le sens résulte de mises en relations internes et externes au texte, bref, de la rencontre d'un contexte et d'un intertexte. La détermination du local par le global s'entend ainsi de deux façons, par l'incidence du texte sur ses parties, et par l'incidence du corpus sur le texte. On pourrait certes objecter ici que la première sorte d'incidence est structurale, en quelque sorte immanente, et la seconde contingente, « imposée de l'extérieur ». Cependant, le texte pointe vers l'intertexte, que ce soit en général par les normes de son genre ou en particulier par des mentions, citations, allusions ou reformulations.

Le rapport à une extériorité, qui fonde conventionnellement le processus d'objectivation, gageait la signification sur la représentation d'une altérité ontologique pleine, celle du monde des objets, et la fondait sur un « réel » qui n'est autre que la doxa des positivistes. Pour la problématique rhétorique/herméneutique, «l'extérieur » du texte est constituée d'autres textes et plus généralement d'autres performances sémiotiques: si, pour objectiver l'interprétation et le sens qui en résulte, le réquisit fondamental d'une altérité est maintenu par la référence au corpus, il n'impose plus le recours à une disparate ontologique, ni à un acte de foi qui subordonnerait l'apparence des signifiés à l'essence des choses.

\section{Vers une sémiotique des performances complexes}

La sémiotique contemporaine a sans doute hérité son déficit herméneutique des sciences du langage, qui, privilégiant le signe, lieu de la référence, et la proposition, lieu de la vérité, répugnent de fait à traiter du texte. Benveniste en témoignait de façon tranchée : "La sémantique, c'est le "sens" résultant de l'enchaînement, de l'appropriation à la circonstance et de l'adaptation des différents signes entre eux. Ça c'est absolument imprévisible. C'est l'ouverture vers le monde. Tandis que la sémiotique, c'est le sens refermé sur lui-même et contenu en quelque sorte en lui-même » (1974 : 21). La frontière de la proposition correspondrait à la démarcation entre ces deux disciplines, ou du moins entre leurs objets. Pour pénétrante qu'elle soit, cette distinction n'exprime qu'un état de fait: la problématique logico-grammaticale prend pour objet les signes et leur combinaison syntaxique qui ne s'étend pas au-delà de la proposition. En revanche, la problématique rhétorique/herméneutique prend pour objet le texte et tous ses paliers, jusqu'à celui du mot. Il est constant que le sens d'un mot dépend du texte où il est inclus; c'est là par exemple une évidence pour la sémantique des textes littéraires.

Le paradigme du signe, propre quant au contenu à la logique et à la philosophie du langage, et, quant à l'expression, à la tradition grammaticale qui culmine dans la morphosyntaxe contemporaine, se trouve ainsi rattaché à la sémiotique, alors que la sémantique se trouve à bon droit associée au paradigme du texte.

En se gardant de généraliser à partir de la linguistique, on doit reconnaître que les textes sont des performances sémiotiques parmi les plus complexes, et exemplaires à ce titre. Par ailleurs, les textes, oraux et écrits, sont des formations plurisémiotiques qui mettent en œuvre outre des langues, des genres et des styles, des systèmes graphiques et typographiques (un signe de ponctuation ne fonctionne pas comme un morphème), prosodiques, gestuels (une kinésique est toujours associée à l'oral). Tous ces aspects restent négligés par la sémiotique, comme par les linguistiques qui se cantonnent à la morphosyntaxe. Enfin et surtout, les relations sémantiques que l'interprétation établit ou reconnaît entre les différentes parties d'un texte sont d'une complexité et d'une variéte 
irréductible à la compositionnalité logique, mais encore mettent souvent sinon toujours en jeu des interprétants qui relèvent d'autres systèmes sémiotiques que les langues.

Dans le domaine de la linguistique, les théories du texte les plus en vue restent rattachées à la problématique logico-grammaticale par deux biais principaux. Le premier résume la textualité à des phénomènes phrastiques qui s'étendent sur des phrases adjacentes (concordances de temps, anaphores) qui sont autant d'isotopies locales. Malgré leur intérêt, les recherches sur la macrosyntaxe et la sémantique de la période ou du paragraphe restent en deçà du texte et de la textualité.

À cette extension de la syntaxe répond une autre voie: celle de la réduction propositionnelle du texte. On en connaît le principe, illustré notamment par Van Dijk : après un codage des phrases en propositions, on supprime les propositions jugées secondaires, pour ne garder enfin qu'une proposition, dite macroproposition, censée représenter le texte ${ }^{7}$. Ce format propositionnel permet la réduction du texte à ce que peut concevoir la problématique logico-grammaticale; aussi a-t-il connu, en psychologie cognitive et en psycholinguistique, un immense succès.

31 Pour sa part, la sémiotique contemporaine ne paraît pas non plus avoir produit de théorie $\mathrm{du}$ texte pleinement compatible avec une problématique rhétorique/herméneutique. Certes, la pratique descriptive des sémioticiens contemporains excède souvent les théories logico-grammaticales dont ils se réclament. Ils ont créé la sémiotique discursive, développé la narratologie pour dépasser ainsi le cadre confiné de la linguistique ${ }^{8}$. Et cependant leurs théories restent gagées sur la signification (propre au signe), non sur le sens (propre au texte). Hjelmslev, en choisissant l'épreuve de la commutation pour définir les unités linguistiques à tous les paliers, a unifié la définition du contenu sur le paradigme du signe (la signification ou dénotation étant définie comme rapport entre une unité du plan du contenu et l'unité correspondante du plan de l'expression). Plus complexe en l'espèce, la théorie de Greimas distingue la signification du sens, mais, par toute une suite de conversions, fait dériver le sens textuel de la structure élémentaire de la signification, emblématiquement résumée à un carré booléen affaibli, dit «carré sémiotique »-qui témoigne encore de l'origine logique du concept de signification'.

Les rapports de la sémantique et de la sémiotique restent ambigus ${ }^{10}$. Mais ce qui nous importe ici, c'est que la sémiotique (dans la mesure où elle se limite aux signes) n'a produit que des théories de la signification, alors que la sémantique (quand du moins elle traite des textes) est appelée à produire des théories du sens.

33 Le texte fait problème pour la sémiotique, dans la mesure où elle est l'héritière de philosophies logiques de la signification, qui s'attachent avant tout à la définition et à la typologie des signes, plutôt que des théories du sens issues de la pratique herméneutique (juridique, religieuse et littéraire notamment). La plus simple manière d'éluder la question consiste à considérer le texte comme un signe ${ }^{11}$. C'est la solution que choisissent Peirce, comme Greimas ${ }^{12}$ ou Eco (cf. 1988: 32 «le Message équivaut au Signe » ${ }^{13}$ ); cette esquive fait évidemment peu de cas de la différence de niveau de complexité entre le signe et le texte, mais surtout empêche de penser l'incidence du global sur le local, en l'occurrence du texte sur chacun des signes qui le composent. En revanche, elle s'accorde parfaitement avec le principe logique (attribué à Frege) de la compositionnalité : comme il pose que la signification d'une expression est composée de la signification de ses sousexpressions, on pourrait dériver le sens du texte de la signification des signes, et l'on annulerait en fait la distinction entre signification et sens. 

à la problématique du texte ${ }^{14}$. Le signe, pourrait-on dire, c'est le contraire du texte.

\section{Vers une sémiotique des cultures}

\section{Pour une praxéologie}

L'ontologie qui a tant pesé sur l'histoire de la sémantique ne s'est-elle pas édifiée sur l'oubli voire le déni de l'action? L'Etre parménidien, unique, identique à lui-même, immobile et invariable, se définit par la négation des caractères fondamentaux de l'action. Et si la connaissance était une action oubliée ? De même qu'une encyclopédie est une archive de passages de textes décontextualisés, une ontologie pourrait être définie comme une archive d'actions: ces «choses» prétendues sont le résultat d'une objectivation dont on oublie qu'elle résulte d'un couplage entre l'individu et son environnement.

Cependant, comme les expériences pédagogiques le confirment, l'appropriation de connaissances passe toujours par une adaptation qui se réalise dans un cours d'action ; les exercices qui permettent cette appropriation n'appliquent pas la théorie, mais la contiennent, pour ainsi dire, «à l'état pratique ». Bref, savoir n'est alors rien d'autre qu'apprendre, au sein d'une pratique sociale.

Comme la théorie et la pratique sont indissociables, l'interprétation et l'action le paraissent également, car toute action est rectification interprétative réitérée d'ellemême. L'interprétation semble alors constituée des moments critiques de l'action, et la théorie interprétative veut présenter la synthèse rationnelle de ces moments critiques à visée régulatrice. L'auto-rectification de l'action dans son cours suppose en effet une distance critique qui trouve un homologue dans la dimension critique de l'herméneutique. Les cercles de la théorie et de la pratique, de l'interprétation et de l'action n'ont au demeurant rien de vicieux, mais témoignent simplement de la dimension herméneutique de la connaissance.

\section{La sémiotique générale et comparée et les sciences herméneutiques}

Les sémiotiques globales sont des philosophies (cf. Locke, Peirce, Apel); sans doute doivent-elles à leur nature philosophique leur caractère global. En revanche, les sémiotiques scientifiques ont des objets régionaux : langues, images, musique, etc.

Une sémiotique générale ne peut être que fédérative; elle définit le champ où la linguistique, l'iconologie, la musicologie et les autres sciences sémiotiques procèdent à leurs échanges pluridisciplinaires.

Si la linguistique se définit comme la sémiotique des langues, la sémiotique discursive se confond avec la linguistique du texte. Cette évidence a été longtemps obscurcie parce que diverses linguistiques restreintes se cantonnaient au palier de la phrase, et déléguaient l'étude de la textualité à diverses disciplines, comme la pragmatique conversationnelle, la poétique ou la sémiotique discursive. Mais une sémiotique discursive autonome ne serait sans doute que "l'envers complice» d'une linguistique restreinte. Outre qu'il faut remembrer les sciences du langage, on ne peut maintenir une frontière disciplinaire 
entre le texte et la phrase. Par exemple, une isotopie est instituée par la récurrence de sèmes (unités microsémantiques du palier inférieur au sémème); et pourtant elle peut s'étendre sur la totalité d'un texte.

Pour rompre avec l'universalisme, il nous faut alors, dans le cadre d'une sémiotique des cultures $^{15}$, édifier une sémiotique historique et comparée. Prenons l'exemple de la narratologie. La méthodologie comparative de Propp procède de la linguistique historique et comparée. Il s'agit, en tenant compte des acquis postérieurs de la narratologie, d'appliquer cette méthodologie à d'autres corpus, pour définir, à l'intérieur des cultures étudiées, les modèles narratifs propres aux divers types de discours, voire aux divers genres. Cette diversification des modèles conditionne une meilleure application des principes méthodologiques généraux de la narratologie, et une meilleure adéquation descriptive.

Plus généralement, il s'agit de rendre compte de diversités, sans confondre les principes théoriques et méthodologiques universels, qui sont ceux de la sémantique (conçue comme branche de la sémiotique des langues), avec tel ou tel modèle théorique, nécessairement partiel quelles que soient ses prétentions à l'universalité. Sans quoi l'on serait conduit à projeter indifféremment sur tout texte la même grille théorique, au risque de lire partout la même chose.

La diversification des modèles sémantiques, qui répond au caractère culturel des textes, permet de renouer les liens avec la philologie, inexplicablement rompus par les courants formalistes, comme avec les autres sciences sociales (histoire, archéologie, etc.) qui participent de la sémiotique des cultures.

\section{La paradoxale objectivité du sens}

L'œuvre exemplaire des grands philologues, comme Spitzer, Auerbach, Bollack, montre la fécondité du projet d'unifier l'herméneutique et la philologie. Étudier les textes au sein d'une sémiotique des cultures ne conduit pas à dissoudre leur étude dans une philosophie de la culture, ni même à étudier les structures culturelles dans leurs manifestations linguistiques. Comme le sens des textes ne leur est pas immanent, il faut pour l'établir tenir compte de leur caractère de formations culturelles. Maintes disciplines participent par vocation et de droit sinon de fait à cette entreprise fédératrice: la littérature comparée, la stylistique, la poétique, la linguistique, mais aussi l'ethnologie et l'histoire.

Cependant, la linguistique et l'ensemble des sciences sociales hésitent depuis leur formation entre les modèles des sciences de la nature, des sciences de la vie et des sciences logico-formelles. Tous les projets réductionnistes s'appuient sur cette hésitation dont il importe de sortir en précisant le mode propre d'objectivité critique de l'objet culturel et les formes de sa temporalité.La fédération des sciences de la culture demande d'ailleurs une conception commune de l'objectivité.

On sait que l'expérience naïve varie sans cesse avec celui qui l'éprouve, et tout l'effort des sciences expérimentales consiste précisément à éliminer ces variations. Le positivisme a tenté de les réduire, ne serait-ce qu'en les négligeant, mais sans succès, car il en demeure toujours un reste que l'on ne peut éliminer. Même dans les sciences de la nature, en physique quantique par exemple, la situation de l'observateur fait partie de la situation expérimentale. Ferdinand Gonseth puis Gilles Cohen-Tannoudji ont employé à ce propos l'image de l'horizon: il appartient à notre champ de vision, qu'il paraît borner. Le réel 
objectif n'en existe pas moins comme ensemble de conjectures : dans un langage unitaire, il reste ce sur quoi nous traçons notre horizon ; et dans un langage infinitaire, il est fait de tous les horizons possibles. Cette situation reste le lot commun de toutes les sciences, leur minimum herméneutique. Mais si dans les sciences de la nature, du moins les sciences physiques, la situation de l'observateur est déterminée par des coordonnées elles-mêmes physiques, repérables dans l'espace-temps, dans les sciences sociales l'espace est médiatisé par la culture, dont la langue, et le temps physique par l'histoire et la tradition. La situation spatio-temporelle de l'observateur est ainsi redoublée par la situation historico-culturelle de l'interprète. Or le linguiste n'est pas seulement un observateur, mais aussi un interprète. La critique philologique joue en quelque sorte le rôle de la méthode expérimentale, non pour éliminer illusoirement toute subjectivité, mais pour hiérarchiser les subjectivités. L'objectivité des sciences de la culture se constitue ainsi dans la reconnaissance critique de leur part de subjectivité ${ }^{16}$.

En disant que le sens du texte est immanent, non au texte, mais à la pratique d'interprétation, nous reconnaissons que chaque lecture, "savante » ou non, trace un parcours interprétatif qui correspond à l'horizon du lecteur. La sémantique des textes propose une description des parcours interprétatifs : le sens actuel du texte n'est qu'une de ses actualisations possibles; le sens "complet» serait constitué de l'ensemble des actualisations, en d'autres termes l'ensemble des horizons possibles.

Comme la notion même de sens complet reste illusoire, une description linguistique ne propose pas une lecture "scientifique» qui se substituerait aux autres, mais une identification des contraintes linguistiques sur les parcours interprétatifs.

Le sens d'un texte n'est clôturable que par l'arrêt de ses lectures, qui appartiennent alors au passé ; il quitte alors ainsi la tradition et la vie, et cette clôture témoigne plutôt d'une fermeture que d'une plénitude, car un livre fermé n'a plus de sens. En revanche, les textes qui sont relus gardent un sens ouvert. Leur sens a une histoire vivante, celle de leur tradition interprétative, série non close de réécritures, qui sont autant de nouvelles lectures : elles dépendent de la pratique où elles prennent place, obéissant à des objectifs éthiques, esthétiques, ou cognitifs. Cependant, le plaisir, le devoir, et la volonté de savoir restent inassouvis. Sur ce point crucial, une sémantique des textes peut distinguer entre les structures closes, qui épuisent la lecture, et les structures ouvertes, qui permettent au lecteur de transformer l'équivoque en infini.

Discerner la spécificité des sciences de la culture permet en outre de dépasser la fausse complémentarité qui distingue les "sciences humaines" et les "sciences sociales", lointain écho peut-être de combats surannés entre l'humanisme et le marxisme.

51 Leur richesse réside dans deux diversités : celle des cultures, qui les fait se mouvoir dans des temps et des espaces différenciés; et, pour chaque objet culturel, la multiplicité des paramètres non reproductibles, qui empêchent toute expérimentation au sens strict et écartent du même coup le modèle des sciences physiques. Même promus au rang d'observables, les faits humains et sociaux restent le produit de constructions interprétatives.

Les sciences de la culture sont les seules à pouvoir rendre compte du caractère sémiotique de l'univers humain. Pour connaître l'humain par l'homme, elles doivent reconnaître la part qu'il prend dans cette connaissance, non seulement comme destinataire critique de " résultats », mais comme acteur doué d'affects et de responsabilité. 


\section{Projet anthropologique et caractérisation} des sexes, âges, tempéraments, nations, etc., avec autant de soin que les sciences naturelles étudient les races et variétés du monde animal. Quoi qu'il ne s'agisse à proprement parler que de savoir combien divers l'homme peut être, il faut faire comme s'il s'agissait de déterminer combien divers est en fait l'homme individuel ${ }^{17}$. Le programme anthropologique de Humboldt connut trois étapes successives : caractériser la différence sexuelle, puis la différence nationale, enfin la différence linguistique, ce qui le conduit à se consacrer, de 1819 à sa mort, à la linguistique comparée. Alors que le nombre de sexes reste fort restreint, regrettablement selon certains, les nations se comptent par centaines, et les langues par milliers. Mais leur diversité ne s'arrête pas là, et Humboldt les envisage dans leur diversité interne, jusqu'à traiter de leurs usages individuels. Ainsi son programme anthropologique va-t-il de l'humanité à l'individu et aboutit à reconnaître autant de langues que d'hommes.

anthropologie de la diversité n'avait jusque là été ébauchée que chez Montaigne, sous une forme d'ailleurs radicale mais sans prétention scientifique: elle trouve chez Humboldt ses fondements épistémologiques et non plus seulement éthiques. Alors que la philosophie du langage est universalisante, la caractérisation des langues suppose que la linguistique décrit leurs singularités sans se cantonner à des règles générales, voire qu'elle reconnaisse dans les lois linguistiques une généralisation de phénomènes singuliers et non répétables, à la différence des phénomènes physiques ${ }^{18}$.

Le programme de caractérisation revêt ainsi une grande portée épistémologique, et Humboldt note ainsi: "Dans le monde inorganique il n'y a pas d'individualité qui pourrait être considérée comme un être existant en soi, et dans le monde organique, les sciences ne descendent jamais jusqu'à l'individu » (GS: VI-150 ; Trabant 1999: 129). En ce sens, le processus de caractérisation est définitoire des sciences de la culture. Corrélativement, l'unicité de l'objet, qui culmine dans l'œuvre d'art non reproductible, peut devenir la caractéristique de l'objet culturel. Enfin, la caractérisation est un processus progressif indéfini : elle peut s'étendre aux parties de l'objet, et conduire par exemple dans l'étude d'un texte à montrer pourquoi tel mot dans tel contexte est un hapax ${ }^{19}$.

56 La portée d'une telle épistémologie de la diversité nous paraît considérable. Comment transformer en point de vue de la diversité les postulats traditionnellement universalistes de la gnoséologie comme de l'épistémologie ${ }^{20}$ ? Jusqu'à la constitution de la linguistique générale, la diversité des langues, voire leurs parentés, étaient connues, mais ne faisaient pas l'objet d'un programme de comparaison, car on se contentait de la ramener à des principes rationnels communs exprimés par des grammaires générales prétendant à l'universel. Elle n'est devenue un problème scientifique qu'à partir du moment où l'on a pu sortir de l'universel, et où leur contingence a pu devenir significative ${ }^{21}$.

57 La mesure de la diversité des cultures a suivi le même chemin que la perception de la diversité des langues. De fait, la découverte, ces deux dernières décennies, de " cultures " animales, notamment chez les primates, suggère que l'innovation et sa transmission ne suffisent pas à définir la spécificité des cultures humaines; la diversification des pratiques techniques et sémiotiques les distingue des « cultures » animales, ce qui érige la 
caractérisation progressive au rang de programme unificateur pour les sciences de la culture.

Sans revenir au débat entre Herder et Kant, rappelons l'enjeu intellectuel de prendre pour objet la diversité culturelle. Comme chez Kant la Raison se développe pleinement dans l'espèce et non dans l'individu, les différences sont tout simplement inessentielles du point de vue de la Raison, d'où l'universalisme et le cosmopolitisme qui en découlent ${ }^{22}$. Mais le concept de cosmopolitisme doit être réélaboré pour limiter l'universalisme qui lui a pourtant donné carrière. La perspective sémiotique peut en effet s'écarter de la philosophie transcendantale en "remplaçant", même comme condition de la connaissance, la Raison par les cultures, et en restituant à la description des objets culturels le caractère critique que la philosophie kantienne avait emprunté à la philologie. Si la Raison peut être pure, une culture ne l'est jamais, car elle est le produit de son histoire. Comparer les cultures et les langues, c'est passer de l'universel au général, c'est aussi passer de l'identité postulée à l'équivalence conquise, du droit au fait, de l'universel au mondial.

Poursuivant un objectif de caractérisation, une sémiotique des cultures se doit donc d'être différentielle et comparée, car une culture ne peut être comprise que d'un point de vue cosmopolitique ou interculturel : pour chacune, c'est l'ensemble des autres cultures contemporaines et passées qui joue le rôle de corpus. En effet, une culture n'est pas une totalité : elle se forme, évolue et disparait dans les échanges et les conflits avec les autres.

La linguistique historique et comparée a acquis sur ce point une expérience à élaborer et à transmettre. L'enjeu est important : comment reconstruire le concept d'humanité hors de la théologie dogmatique et de la biologie qui rivalisent de déterminisme? Comment concevoir l'humanité à partir des humanités - en comprenant par là, aussi, les sciences sociales?

\section{Médiations}

61 La place du monde sémiotique, en position médiatrice chez l'homme entre le monde physique et le monde des (re)présentations, détermine la fonction épistémologique de la sémiotique elle-même. Nous avons pris le parti d'une sémiotique des cultures, et non celui des sémiotiques universelles ou transsémiotiques, sortes de philosophies du sens oublieuses de leur dimension réflexive.

Quelques précisions terminologiques s'imposent ici. Nous avons utilisé deux expressions, sciences de la culture et sémiotique des cultures, dont aucune ne convient parfaitement à notre propos : alors la première est empruntée à Cassirer (1991 [1936-1939]), la seconde renvoie implicitement à l'École de Tartu. Le troc des pluriels que souligne leur juxtaposition permet de poser deux questions : une ou plusieurs sciences? la culture ou les cultures?

Pour Cassirer, sciences de la culture s'oppose implicitement à sciences de la nature, et cette opposition se superpose à celle que Dilthey a tracée et les sciences de l'esprit ( Geisteswissenschaften) entre les sciences de la nature (Naturwissenschaften); cependant Cassirer transpose implicitement en culture l'esprit selon Dilthey. La réflexion y gagne, car, même si l'allemand distingue l'intellectuel, geistig, et le spirituel, geistlich, le terme forgé par Dilthey témoignait d'un spiritualisme individualisant qui ne pouvait véritablement aider à fédérer un champ scientifique. 

critique reste à élaborer. La sémiotique peut sans doute y contribuer, mais la sémiotique des cultures contemporaine est-elle en mesure de le faire? Pour l'école de Tartu, l'expression sémiotique des cultures (ou de la culture) vient sans doute de la littérature comparée - le domaine de son principal animateur Iouri Lotman. Cependant, cette sémiotique des cultures, devenue culturologie, a remplacé dans les cursus d'enseignement russes le Diamat - ou matérialisme dialectique -, en conservant souvent les mêmes professeurs. Elle est parfois tentée de remplacer la dialectique de classes antagonistes par celle de cultures antagonistes, ce qui ne va pas sans quelques risques de dérives nationalistes. Les mentalités seraient formées par la langue et la nation, donc un nonrusse ne pourrait en fait comprendre un texte russe - ce qui rappelle fort certaines thèses de Heidegger voire de Gadamer sur le Grund, fond d'appartenance national et traditionnel qui conditionnerait toute compréhension. En outre, chaque culture est présentée comme une monade assiégée, qui n'aurait à l'égard des autres que surestimation ou répulsion; cette ambivalence, fréquente dans l'histoire intellectuelle russe, ne saurait être érigée en définition de la culture.

L'expression sémiotique des cultures renvoie-t-elle à une seule science ou à plusieurs ? Dans le premier cas, on penchera vers une anthropologie philosophique, celle par exemple de Cassirer dans son Essai sur l'homme: un tel champ de réflexion, fort nécessaire, ne peut évidemment se prétendre une science. Dans le second cas, on cherchera à restituer l'unité des sciences humaines, et la réflexion sur le sémiotique en tant que domaine scientifique, plutôt que sur la sémiotique en tant que science. En effet, l'omniprésence des signes rend pour ainsi dire impossible la constitution de la sémiotique comme discipline. Une sémiotique des cultures, notamment, ne serait-elle pas une science des sciences? La sémiotique des cultures n'est pas à vrai dire une discipline, mais le projet même de redéfinir la spécificité des sciences humaines et sociales: les cultures embrassent la totalité des faits humains, jusqu'à la formation des sujets. Elles restent cependant difficiles à concevoir, faute précisément d'un point de vue sémiotique sur la culture. En d'autres termes, c'est la reconnaissance de la spécificité et de l'autonomie relative du monde sémiotique qui permet de délimiter le champ des sciences de la culture, et d'en finir avec le dualisme traditionnel qui commande la division proposée par Dilthey.

68 Le projet saussurien d'une sémiologie naît de la volonté de définir l'ordre scientifique auquel appartient la linguistique : «On a discuté pour savoir si la linguistique appartenait à l'ordre des sciences naturelles ou des sciences historiques. Elle n'appartient à aucun des deux, mais à un compartiment des sciences qui, s'il n'existe pas, devrait exister sous le mot de sémiologie [...] le système sémiologique 'langue' est le seul [...] qui ait eu à affronter cette épreuve de se trouver en présence du Temps, qui ne se soit pas simplement fondé de voisin à voisin par mutuel consentement, mais aussi de père en fils par impérative 
tradition, et au hasard de ce qui arriverait en cette tradition, chose hors de cela inexpérimentée, non connue ni décrite » (1974: II-47).

S'il est clair ici que la sémiotique est conçue comme un compartiment des sciences et non comme une discipline de plus, la notion de Temps traditionnel, distingué de fait du temps historique, mérite une grande attention. Les performances sémiotiques se déploient dans le temps de la tradition, forme de temporalité propre aux objets culturels qui ne se confond ni avec le temps physique ni avec le temps de l'histoire ${ }^{23}$. Si les sciences naturelles se satisfont du temps darwinien de l'évolution, les sciences de la culture se meuvent dans un temps lamarkien, fait de traditions et de ruptures. Ce temps traditionnel n'obéit pas aux métriques du temps historique : ni régulier, ni connexe, ni déterministe, il laisse ouvertes des rétrospections, des anticipations, il met en contact les contemporains et les anciens, les proches et les étrangers. L'herméneutique et la philologie nous permettent ensemble d'approcher ce temps interne du monde sémiotique.

70 Entre le temps physique et le temps traditionnel, le temps historique occupe une position doublement intermédiaire : c'est à la fois le temps externe du couplage des sociétés avec leur environnement et celui de leur auto-réflexion, où elles choisissent ce qui fait événement pour elles.

71 Les formulations du projet de la sémiotique des cultures sont restées éparses chez divers auteurs et elle ne s'est pas constituée en discipline autonome. En effet, elle garde une vocation épistémologique: fédérer les sciences de la culture autour des concepts de langage et d'interprétation, déplacer l'opposition métaphysique entre le sujet et l'objet en une distinction relative entre l'interprétation et le signe (le signe est une interprétation objectivée et stabilisée dans son prétexte signifiant), restituer la complexité radicale des textes et autres performances sémiotiques, sans chercher à les unifier dans une totalité. Comme une totalité se définit par l'unité à soi, elle n'a pas de sens, puisque le sens est fait de différences irréductibles reconnues et qualifiées par les parcours interprétatifs: la sémiotique des cultures se trouve donc devant la nécessité constitutive de rompre avec les ontologies, tant celle des sciences de la nature que celle des sciences logico-formelles.

72 Elle s'ouvre ainsi d'une part sur l'éthologie des sociétés humaines, de l'autre sur une philosophie des formes symboliques. Face aux programmes réductionnistes qui menacent l'ensemble des sciences de la culture, son développement reste un enjeu pour les années à venir. D'une part, la sémiotique des cultures semble la seule perspective globale qui puisse s'opposer au computationnalisme qui a hypothéqué la problématique et les résultats des recherches cognitives. D'autre part, comprendre la médiation sémiotique reste indispensable pour décrire les facteurs culturels dans la cognition, jusqu'ici gravement sous-estimés par les recherches cognitives. Pour culturaliser les sciences cognitives, il faudrait en outre reconnaître le caractère culturellement situé de toute activité de connaissance, l'activité scientifique comprise.

73 Le culturel s'identifie ici à l'humain, car la médiation sémiotique, caractéristique de la cognition humaine, la définit sans doute comme telle. Ainsi s'ouvre l'espace d'une réflexion sur la genèse des cultures, liée évidemment à la phylogenèse, mais échappant à des descriptions de type néo-darwinien. La distinction des formes symboliques, la diversification des langues, celle des pratiques sociales, celle des arts, tous ces processus poursuivent l'hominisation par l'humanisation, mais ne s'inscrivent pas dans la longue durée, s'autonomisent à l'égard du temps de l'espèce, et conditionnent la formation du temps historique sans pourtant se laisser rapporter à ses rapides scansions. 


\section{BIBLIOGRAPHIE}

Agamben G. 1998 : Stanze, Paris, Payot.

Benveniste É. 1974 : Problèmes de linguistique générale, tome II, Paris, Gallimard.

Bréal M. 1897 : Essai de sémantique, Paris, Hachette [rééd. Brionne, Gérard Monfort, 1982].

Cassirer E. 1972-1975 : La philosophie des formes symboliques, Paris, Minuit, 3 vol.

- 1991 [1936-1939] : Logique des sciences de la culture, Paris, Cerf.

De Mauro T. 1971 : Senso e Significato, Bari, Adriatica.

Ducrot O. \& Todorov T. 1972 : Dictionnaire encyclopédique des sciences du langage, Paris, Le Seuil.

Dumarsais C. C. 1988 [1730] : Traité des tropes, éd. F. Douay-Soublin, Paris, Flammarion.

Eco U. 1975 : Trattato di semiotica generale, Milan, Bompiani.

- 1989 : Sémiotique et philosophie du langage, Paris, P.U.F.

- 1992 : Le signe, Paris, Gallimard.

Frege G. 1971 : Écrits logiques et philosophiques, Paris, Le Seuil (trad. C. Imbert).

Greimas A. J. 1966 : Sémantique structurale, Paris, Larousse.

- 1970 : Du Sens, Paris, Seuil.

- 1976 : Maupassant. La sémiotique du texte, Paris, Seuil.

— \& Courtés J. 1979 : Sémiotique. Dictionnaire raisonné de la théorie du langage, Paris, Hachette.

Hjelmslev L. 1971 : Essais linguistiques, Paris, Minuit.

Humboldt W. von 1903-1936 : Gesammelte Schriften, Berlin, Behr, 17 vol.

- 1974 : Introduction à l'œuvre sur le kavi, Paris, Seuil.

- 2000 : Sur le caractère national des langues et autres écrits sur le langage, Paris, Le Seuil [introduit et traduit par Denis Thouard].

Jacob P. 1998 : Pourquoi les choses ont une signification, Paris, Odile Jacob.

Johnson-Laird P. N. 1988 : « La représentation mentale de la signification », RISS, 115 : 53-69.

Katz J.J. 1971 : La philosophie du langage, Paris, Payot.

Linsky L. 1974 : Le problème de la référence, Paris, Le Seuil.

Lotman I. 1999 : La sémiosphère, Limoges, PULIM.

Lyons J. 1978 : Éléments de sémantique, Paris, Larousse.

- 1980 : Sémantique linguistique, Paris, Larousse.

Malrieu D. \& Rastier F. 2001 : « Genres et variations morphosyntaxiques », Traitements

automatiques du langage, 16.

Minsky M. 1975 : « A framework for representing knowledge », in Winston P. (éd.) 1975 : The

Psychology of Computer Vision, New York, McGraw Hill : 211-280. 
Montague R. 1974 : Formal Philosophy, New Haven (Conn.), Yale University Press.

Mounin G. (éd.) 1974 : Dictionnaire de la linguistique, Paris, P.U.F.

Ogden C.K. \& Richards I. A. 1923 : The Meaning of Meaning, Londres, Routledge and Kegan Paul.

Pap L. 1990 : Semiotics. An integrative survey, Toronto, monograph of the Toronto Semiotic Circle.

Pollock J-Y. 1997 : Langage et cognition, Paris, P.U.F.

Propp V. 1965 [1928] : La morphologie du conte, Paris, Le Seuil.

Peirce C. S. 1956 : Collected Papers, Harvard, Harvard University Press, 6 vol.

Rastier F. 1987 : Sémantique interprétative, Paris, P.U.F.

- 1989 : Sens et textualité, Paris, Hachette.

- 1991 : Sémantique et recherches cognitives, Paris, P.U.F.

-, Cavazza M. \& Abeillé A. 1994 : Sémantique pour l'analyse, Paris, Masson.

- 1995a : «Communication ou transmission?», Césure, 8 : 151-195.

- 1995b : «La sémantique des thèmes - ou le voyage sentimental », in Rastier F. (éd.) 1995 :

L'analyse thématique des données textuelles - L'exemple des sentiments, Paris, Didier : 223-249.

- 1996a : «Représentation ou interprétation? - Une perspective herméneutique sur la médiation sémiotique », in Rialle V. \& Fisette D. (dir.) 1996 : Penser l'esprit : des sciences de la cognition à une philosophie cognitive, Grenoble, Presses Universitaires de Grenoble : 219-239.

- 1996b : «Pour une sémantique des textes - Questions d'épistémologie », in Rastier, F. (éd.)

1996 : Textes et sens, Paris, Didier : 9-38.

- 1996c : « Problématiques du signe et du texte », Intellectica, $23: 11-53$.

- 1997 : « Herméneutique matérielle et sémantique des textes », in Salanskis J-M., Rastier F. \& Scheps R. (éds.) 1997 : Herméneutique : textes, sciences, Paris, P.U.F.

-2001 : Arts et sciences du texte, Paris, P.U.F.

Saussure, F. de 1968-1974 : Cours de linguistique générale, Wiesbaden, Harrassowitz, 2 tomes [édition critique Rudolf Engler].

- 1972 [1916] : Cours de linguistique générale, Paris, Payot [édition Tullio de Mauro].

- 1986 : Le leggende germaniche, Este, Zielo [édition Anna Marinetti \& Marcello Meli].

- 2001 : Écrits de linguistique générale, Paris, Gallimard (= « Bibliothèque de philosophie »), [édition Simon Bouquet \& Rudolf Engler].

Sowa J. 1984 : Conceptual Structures: Information processing in mind and machines, New York, Addison-Wesley.

Trabant J. 2000 : Présences de Humboldt, Paris, Maison des sciences de l'homme.

Wittgenstein L. 1961 : Tractatus logico-philosophicus, Paris, Gallimard.

\section{NOTES}

1. Parmi les agents de la sémiosis, Peirce mentionne des animaux comme le «chameleon and many kinds of insects » (MS 318: 205-206), les microorganismes, "a little creature » sous un 
microscope (CP 1.269), les plantes qui utilisent des signes («plants that make their living by uttering signs, and lying signs", MS 318: 205-206). Mieux la sémiosis, et avec elle la pensée «thought, » advient même « in crystals, and throughout the physical world » (CP 4.551).

2. Les y réduirait-on, il faudrait encore reconnaître que personne n'a jamais été capable de dresser une liste finie des signes d'une langue vivante.

3. Empirique est entendu dans l'acception que définissait Althusser quand il opposait l'objet empirique et l'objet de connaissance. Nous posons ici que le texte est tout à la fois l'objet empirique de la linguistique et son objet de connaissance.

4. Cf. Rastier 1996a.

5. Nous avons détaillé par ailleurs, en étudiant les relations sémantiques en contexte, les analogies entre le traitement des contrastes en perception visuelle et auditive et en perception sémantique (cf. Rastier 1991, ch. VIII).

6. Peut-être la référence logique n'est-elle en fait qu'une objectivation de la référence philologique, ce pourquoi on a pu affirmer que l'ordre herméneutique domine l'ordre référentiel. Par exemple, la référence de la Cousine Bette n'est pas « directement » la France louis-philipparde, mais en premier lieu sinon exclusivement La Comédie Humaine, augmentée des romans d'Eugène Sue que Balzac voulait surpasser.

7. Au début des années soixante, Ruwet avait montré la voie en résumant un sonnet de Louise Labé à la macroproposition Je t'aime. La valeur caractérisante de ce genre de description semble faible, car des milliers d'autre sonnets de l'époque auraient bien entendu conduit au même résultat.

8. Naguère constituées en opposition à des théories linguistiques restreintes à la morphosyntaxe, les théories sémiotiques les plus connues considèrent le niveau linguistique comme une variable «de surface». Avec l'extension du champ des études linguistiques, le principe d'une sémiotique discursive autonome devient de plus en plus difficile à défendre.

9. Le parcours génératif produit le sens textuel, qui appartient aux structures linguistiques « de surface ", à partir de la structure élémentaire de la signification, érigée en modèle constitutionnel, donc par définition le plus "profond" qui soit, de toute sémiotique. Pour ma part, je considère simplement cette structure élémentaire comme une des structures attestées parmi les classes lexicales simples.

10. Par exemple, la sémiotique de Hjelmslev a précédé sa sémantique structurale (1957); mais la sémiotique de Greimas, présentée dans Du Sens en 1970, est issue de sa Sémantique structurale (1966).

11. Un grand apport de Hjelmslev aura été de rompre avec la sémiotique du signe, léguée par une philosophie du langage issue de la métaphysique aristotélicienne, pour lui substituer une sémiotique des figures et des procès.

12. Cf. «Le texte se présente comme un signe, dont le discours, articulé en isotopies figuratives multiples, ne serait que le signifiant invitant à déchiffrer son signifié » $(1976$ : 267).

13. Cf. aussi : « le macrosigne qu'est le Rouge et le Noir peut être vu comme l'interprétant de la proposition Napoléon est mort le 5 mai $1821 »$ (ibid.).

14. Cf. Rastier 1996 a.

15. Cf. Rastier 1991.

16. Cf. cette réflexion de Szondi, à propos de la philologie, mais qui convient à toute science sociale tentée par le modèle des sciences de la nature: "Dès qu'elle tente de mettre entre parenthèses le sujet connaissant au nom d'une prétendue objectivité, elle court le danger de dénaturer, en recourant à des méthodes inappropriées, des faits empreints de subjectivité, sans être en mesure de percevoir son erreur » $(1981: 15)$.

Un autre aspect de la distance critique, reste très mal compris, et il importe de réhabiliter l'ironie : «Que l'ironie romantique, avec les Schlegel justement, ait pu donner lieu à une attitude authentiquement philologique et scientifique (avec notamment l'impulsion déterminante donnée 
à la linguistique indo-européenne) est un phénomène que l'on n'a pas suffisamment interrogé dans la perspective d'une fondation critique des sciences humaines)» (Agamben 1998: 9). L'ironie distingue sans doute les savants - dont nous manquons - des scientifiques ordinaires.

17. Plan d'une anthropologie comparée, GS : I-390 .

18. En transposant une distinction husserlienne, on peut suggérer que les sciences exactes sont nomothétiques, et que les sciences rigoureuses sont idiographiques. Les sciences de la culture sont rigoureuses, dans la mesure où elle témoignent de l'exigence critique.

19. Le programme de caractérisation s'applique ainsi aux langues, comme le résume Denis Thouard: "Etant donné une structure linguistique particulière, le caractère est l'effet des transformations induites au cours de l'histoire par les locuteurs dans leur usage de la langue et déposée en elle. Rien n'est fixe en effet, des habitudes s'inscrivent peu à peu dans la langue, non seulement au plan sémantique et phonétique, mais dans l'organisation syntaxique elle-même. Les langues ne sont pas données une fois pour toutes, de nouvelles peuvent émerger, d'autres disparaître. [...] Le caractère d'une langue est ainsi le précipité des actes de discours en tant qu'ils se sont déposés dans la langue. La linguistique humboldtienne s'intéresse à ce titre aux performances individuelles des locuteurs, dont la littérature enregistre les innovations. [...] Le concept de "caractère", au sens précis où l'emploie Humboldt, permet ainsi d'articuler les approches synchroniques et diachroniques du langage. En s'attachant aux œuvres, il permet d'envisager le développement d'une linguistique textuelle, intégrant dans son champ les réalisations singulières du discours » (Thouard $2000: 170)$.

20. Depuis la redécouverte au XIII ${ }^{\mathrm{e}}$ siècle des Seconds analytiques, la thèse aristotélicienne qu'il n'y a de science que du général a été interprétée comme un principe d'universalité.

21. Les grammaires universelles qui ont rivalisé au cours du $\mathrm{XX}^{\mathrm{e}}$ siècle ont continué le programme médiéval et classique (cf. Chomsky se revendiquant de Port-Royal): de fait, caractériser la diversité des langues n'est pas de leur ressort. En présentant le programme minimaliste chomskyen, Pollock écrit par exemple : « les langues nationales comme le français, le chinois et l'italien ne sont donc pas directement du ressort de la linguistique : en effet, ce ne sont pas des réalités psychologiques / neurophysiologiques individuelles, mais des entités historiques, politiques et sociologiques, comme les nations qui leur correspondent parfois » (1997, p.11).

22. Cf. Idée d'une histoire universelle d'un point de vue cosmopolitique, 1784. [tr. fr. L. Ferry, CEuvres, Bibliothèque de la Pléiade, t. II].

23. Il faudrait ici relire ces propos de Saussure: "nous constatons tout de suite l'entière insignifiance d'un point de vue qui part de la relation d'une idée et d'un signe hors du temps, hors de la transmission, qui seule nous enseigne, expérimentalement, ce que vaut le signe » (1968: I-273).

\section{RÉSUMÉS}

À la différence de la sémiotique du signe, extension de la tradition logico-grammaticale, le programme saussurien d'une « science des signes au sein de la vie sociale » conduit à privilégier les textes et autres performances sémiotiques complexes, comme à les contextualiser au sein des pratiques de production et d'interprétation où ils prennent sens. Cela permet d'esquisser un programme fédérateur pour les sciences de la culture : il développe l'anthropologie qui a donné naissance chez Humbolt à la linguistique historique et comparée. Après avoir interrogé le statut scientifique de la sémiotique, on souligne qu'une sémantique des textes peut contribuer à 
renouveler sa problématique. La linguistique est en effet la sémiotique des langues, et relève en cela d'une sémiotique des cultures.

Saussure's semiology (« science des signes au sein de la vie sociale »), unlike traditional thinking about signs, which is involved in logic and grammar, induces to give priority to texts and other complex semiotic practices. Hence we put forward that a program for cultural sciences, restoring Humboldt's anthropology, is now to be stretched. Therefore linguistics, which can be said language's semiotics, will become, thus renewed by the study of texts, a part of such a semiotic science of cultures.

\section{AUTEUR}

\section{FRANÇOIS RASTIER}

Modyco - CNRS,e2@ext.jussieu.fr 